\title{
Laparoscopic Cytoreductive Surgery with Hyperthermic Intraperitoneal Chemotherapy (L-CRS/HIPEC) for Perforated Low-Grade Appendiceal Mucinous Neoplasm (LAMN II)
}

\author{
Ed Parkin, MD ${ }^{1,2}$, Chelliah Selvasekar, MD, FRCS ${ }^{1,2}$, Malcolm Wilson, MD ${ }^{1,2}$, Andrew Renehan, PhD, FRCS ${ }^{1,2}$, \\ Sarah O'Dwyer, $\mathrm{MD}^{1,2}$, and Omer Aziz, MD ${ }^{1,2}$ \\ ${ }^{1}$ The Christie NHS Foundation Trust, Colorectal and Peritoneal Oncology Centre, Manchester, UK; ${ }^{2}$ Manchester Cancer \\ Research Centre, University of Manchester, Manchester, UK
}

\begin{abstract}
Introduction. Cytoreductive surgery with hyperthermic intraperitoneal chemotherapy (CRS/HIPEC) is an established treatment for pseudomyxoma peritonei resulting from a perforated low-grade appendiceal mucinous neoplasm (LAMN II). In patients with localized disease, a laparoscopic procedure (L-CRS/HIPEC) can be undertaken.
\end{abstract}

Methods. This video demonstrates L-CRS/HIPEC in a 66-year-old male who had previously undergone an appendicectomy for an LAMN II lesion. The preoperative computed tomography (CT) scan suggested disease localized to the right iliac fossa. However, laparoscopic assessment unexpectedly revealed disease in the pelvis and on the right hemidiaphragm and liver surface.

Results. A technique for treating the thin film of mucin in the pelvis and on the right hemidiaphragm is demonstrated. The liver is mobilized to facilitate ablation of mucin on the serosal surface of the right lobe. Tips and tricks for starting the omentectomy, dealing with the vascular pedicle, and completing the dissection in the left upper quadrant are shown. The Peritoneal Cancer Index (PCI) score was 5 (3 for the right upper quadrant, 1 for the pelvis, 1 for the small bowel), and the cytoreduction score was CC-1. The operative duration was $8.5 \mathrm{~h}$, and length of hospital stay was 5 days. The patient returned to work after 6 weeks.

Discussion. L-CRS/HIPEC can be performed when patients are unexpectedly found to have disease, provided the appendiceal pathology is low grade and the PCI score is low. There are potential benefits to this approach, with a shorter length of hospital stay and faster functional recovery when compared with traditional open surgery.

OPEN ACCESS This article is distributed under the terms of the Creative Commons Attribution 4.0 International License (http:// creativecommons.org/licenses/by/4.0/), which permits unrestricted use, distribution, and reproduction in any medium, provided you give appropriate credit to the original author(s) and the source, provide a link to the Creative Commons license, and indicate if changes were made.

Publisher's Note Springer Nature remains neutral with regard to jurisdictional claims in published maps and institutional affiliations.
Electronic supplementary material The online version of this article (https://doi.org/10.1245/s10434-019-07277-z) contains supplementary material, which is available to authorized users.
(C) The Author(s) 2019

First Received: 3 October 2018; Published Online: 15 April 2019

O. Aziz, MD

e-mail: Omer.Aziz@christie.nhs.uk 